Although 9 patients had a minor rise infaecal calprotectin of $>200$. None of these patients had othersignificant pathology such as inflammatory bowel disease or colonic cancer. Patients reported watery diarrhoea in 54 of 55 cases with weight loss and abdominal pain being described in 16 and 19 patients respectively. Average length of symptoms at time of diagnosis was 4.8 months. 43 patients were on at least one medication which is known to be associated with MC and of these medications were reviewed in 31 cases $(72 \%)$ with culprit medications being stopped or changed in 16 patients (37\%). In total 32 patients were commenced on therapy for their MC with 19 (59\%) receiving a reducing course of Budesonide, 7 (22\%) receiving a fixed dose of Budesonide and 6 (19\%) receiving 5-ASA. All 55 patients were triaged to have 'direct-to-test' colonoscopy, however, only 12 patients (22\%) were referred directly to gastroenterology from GP. In total 42 patients were referred to surgical specialties with 30 of these patients receiving onwards referral to gastroenterology, often via their GP which resulted in a delay of up to 12 months in some patients. Only 5 patients had recurrent disease, with 2 patients undergoing active investigation at the time of data analysis.

Conclusion Our data set is in keeping with other published data on MC. There is a variation in both referral pathway and management of these patients in FVRH. There exists no common pathway and time taken to see a gastroenterologist is often lengthy and arduous. Review of culprit medications appears to be often overlooked and this may result in ongoing symptoms or recurrent disease. Within FVRH we are currently undertaking a service change with an aim to streamline the referral process, standardise management and improve the patient journey.

\section{PWE-042 PREDICTING THE RISK OF GASTRO-INTESTINAL CANCER IN IRON DEFICIENCY ANAEMIA}

1,20rouba Almilaji*, ${ }^{1}$ Peter Thomas, ${ }^{2}$ Jonathon Snook. ${ }^{1}$ Bournemouth University, UK; ${ }^{2}$ Poole Hospital NHS Foundation Trust, UK

\subsection{6/gutjnl-2019-BSGAbstracts.366}

Introduction Iron deficiency anaemia (IDA) is a common clinical presentation, and in a significant minority of cases $(-0 \%)$ is the first indication of an underlying cancer in the gastrointestinal (GI) tract. IDA is therefore considered an indication for fast-track endoscopic investigation, though the majority of cases will not actually have cancer. This study explores whether cancer risk in IDA can be predicted on the basis of simple and objective clinical variables.

Method A study of the predictive value of sex, age, haemoglobin concentration $(\mathrm{Hb})$, mean red cell volume (MCV) and iron studies for the risk of GI malignancy on subsequent investigation in adults with confirmed IDA attending a single IDA clinic. The study population comprised a training dataset $(\mathrm{n}=2295)$ and a validation dataset $(\mathrm{n}=602)$. The analysis was undertaken using logistic regression, and an App to predict the probability of GI cancer in IDA was developed as a clinical tool using $\mathrm{R}$ Shiny programming language.

Results Using the training data, the best model showed that the risk of GI malignancy was strongly associated with sex (OR for males: 2.83, $\mathrm{P}<0.001)$ age, (OR: 1.05 for each added year, and $\mathrm{Hb}(\mathrm{OR}$ : 0.975 for each $\mathrm{g} / \mathrm{l}$ fall, $\mathrm{P}<0.001)$ see figure 1 for the combined effects with their confidence intervals. GI cancer risk was less strongly associated with

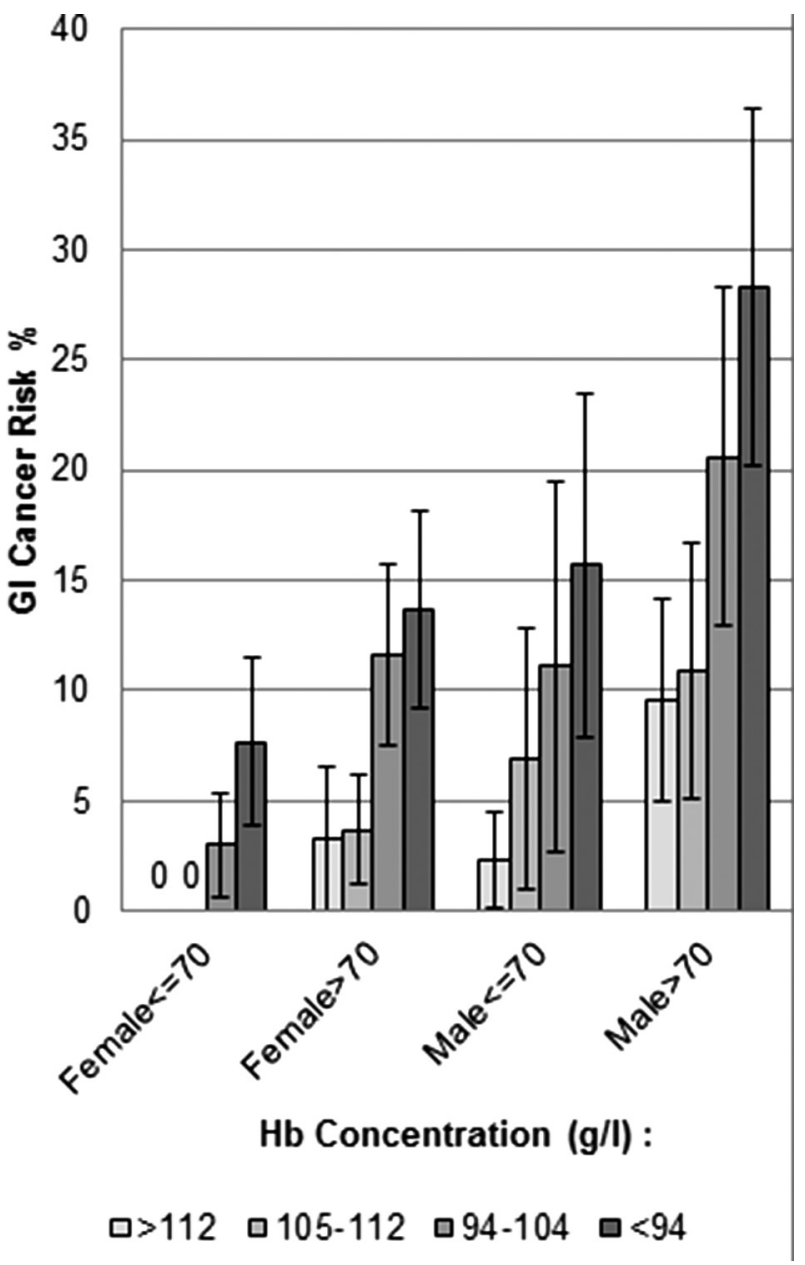

Abstract PWE-042 Figure 1

MCV (OR: 0.971 for each fl fall, $\mathrm{P}<0.05$ ), with a complex relationship largely due to an increased cancer risk in those with more severe anaemia, particularly in younger age-groups.

The model was tested on the validation data and produced similar results. It allowed stratification of $13 \%$ of the study population into a sub-group at high risk of cancer (arbitrarily defined as $>15 \%), 28 \%$ into a sub-group at low risk (-\%), and $16 \%$ into a sub-group at very low risk $(<1 \%)$.

Conclusion This study confirms that a simple clinical scoring system can effectively stratify patients with IDA according to GI cancer risk, allowing stretched investigational resources to be targeted at the high-risk group, whilst perhaps avoiding invasive investigation altogether in those predicted to be at extremely low risk. The App developed has the potential to provide a quick estimate of GI cancer risk in clinical settings, and so facilitate patient counselling.

\section{PWE-043 BURDEN OF CLOSTRIDIUM DIFFICILE INFECTION ON HOSPITALIZATION AND ITS IMPACT ON SPECIFIC MAJOR DIAGNOSTIC CATEGORIES}

${ }^{1}$ Diego Caroli ${ }^{*},{ }^{2}$ Mario Saia, ${ }^{1}$ Erik Rosa-Rizzotto, ${ }^{2}$ Claudio Pilerci, ${ }^{1}$ Franca De Lazzari. ${ }^{1}$ St. Anthony Hospital, Padova, Italy; ${ }^{2}$ Azienda Zero, Padova, Italy

\subsection{6/gutjnl-2019-BSGAbstracts.367}

Introduction Clostridium difficile infection (CDI) has emerged as a major healthcare-associated infection, with an important 
and constant increase in CDI hospitalization and mortality rates.

Methods To describe the epidemiology of CDI hospitalization in Veneto Region, a retrospective analysis based on the anonymous regional database was carried out including all discharges of the period 200-016 of population over 65 years of age with a code for CDI (008.45) as principal or secondary diagnosis.

Data were stratified by Major Diagnostic Categories (MDC), which are formed by dividing all ICD-9-CM diagnoses into 25 mutually exclusive diagnosis areas, to evaluate the in-hospital mortality risk attributable to CDI of each MDC.

Results 6640 discharges with CDI in patients over 65 years of age were tracked, with an overall lenght of stay (LOS) of $25.6 \pm 22,7$ days. Hospitalization distribution by gender showed a prevalence of females $(63.2 \%)$ characterized by a higher mean age $(80.2 \pm 14.6$ Vs. $75.6 \pm 16.4$ yrs;p<0.001). The rate of CDI diagnoses per 100000 overall admissions increased steeply with age, and reached a peak of 222.4 every 100000 discharges among the very elderly ( $>$ 85years). In the frame of over 65 years patients the age class distribution showed a higher risk in $7-4$ (OR:1.62; $<0.001)$ and an almost double in $>85$ years $(\mathrm{OR}: 2.1 ; \mathrm{p}<0.001)$ respect to $6-4$ age class. Over 65 years of age patients admission with CDI diagnosis represent $2.1 \%$ of overall ordinary admission and cause to the LOS excess of 15.7 days and represent the 5.4\%o of overall hospitalization days. Also in-hospital mortality rate showed an excess risk attributable to CDI (OR:1.91;p<0.001). As expected the MDC with most discharges of CDI was n.6 "Digestive"(36\%) followed by 4 "Respiratory" (17\%). The higher excess of in-hospital mortality risk was a prerogative of n. 8 "Musculoskeletal"(OR:5.55;p<0.001) followed by 11 "Kidney And Urinary Tract" (OR:3.6;p<0.001) and 5 "Circulatory"(OR:2.35; p<0.001). Otherwise the presence of CDI showed a slight excess risk in MDC n.6 "Digestive"(OR:1.03; $\mathrm{p}<0.05), 18$ "Infectious and Parasitic"(OR:1.05;p<0.001) and 4 "Respiratory"(OR:1.01; $<0.001)$.

Conclusion In the observation period the standard hospitalization rate for CDI was almost doubled and standard of mortality rate even increased sixfold, with an increase in their frequency, as well as in their severity and in the additional days of hospitalization.

Moreover the stratification of CDI by MDC showed a serious scenary in patients affected by musculoskeletal, kidney and circulatory diseases with an excess of in-hospital mortality risk associated to CDI.

\section{PWE-044 ACUTE COLON DIVERTICULITIS HOSPITALIZATION TRENDS IN VENETO REGION (NORTHEAST ITALY)}

${ }^{1}$ Mario Saia, ${ }^{1}$ Diego Caroli*, ${ }^{1}$ Erik Rosa-Rizzotto, ${ }^{2}$ Claudio Pilerci, ${ }^{2}$ Franca De Lazzari. ${ }^{1}$ St. Anthony Hospital, Padova, Italy; ${ }^{2}$ Azienda Zero, Padova, Italy

10.1136/gutjnl-2019-BSGAbstracts.368

Introduction Diverticular disease (DD) of the colon represents a common clinical condition affecting with high rate the population in developed countries. The rates of hospital admissions for Acute Colon Diverticulitis (ACD), the most important complication of DD, is progressively increased. The aim of our study is to describe the hospitalization trend for ACD in Veneto region, including in-hospital mortality, and the impact of specialistic admission hospital units as Gastroenterologic (GE) units.

Methods Data were obtained from the Veneto Region anonymous Hospital Discharge Record database from 2000 to 2017, in which diverticulitis of colon [ICD-9-CM code 562.11 and 562.13 (diverticulitis with and without mention of haemorrhage)] was the primary diagnosis.

Standardized Hospitalization Rate (SHR) per five-year group was calculated and expressed per 100,000 population. To evaluate the impact of admission hospital units on the outcome of ACD management we analyzed in-hospital mortality, need for surgery (NFS) and lenght of stay (LOS).

Results From 2000 to 2017 a total of 33,249 hospitalization for $\mathrm{AD}$ were registered with an increasing rate from 0.30 to 0.46 for 1,000 inhabitants $\left(\mathrm{X}^{2}\right.$ trend:514.182, $\mathrm{p}<0.001$; mean increase of $2.6 \%$ per year,p $<0.001)($ FIG.1). Mean age was 66.1 \pm 28.4 , higher in female $(70.1 \pm 29.8$ Vs.60.5 \pm 24.9 ; $\mathrm{p}<0.05$ ) and the higher hospitalization rate (overall mean:0.39) was in female (0.43Vs.0.34;OR:1.30;CI95\%:1.2$.32 ; \mathrm{p}<0.001)$. SHR increased with age reaching the higher value in patients $\geq 85$ years (162.43) with an increase of hospitalization over the study period for all class ages $(p<0.001)$. Female showed an higher prevalence of hospitalization compared to men (OR:1.30;CI95\%: 1.2-.32;p<0.001), and both genders presented an increasing trend, with a mean increase per year of $1.9 \%$ and $3.8 \%$ respectively $(p<0.001)$. NFS was substantially stable (19.4\%) while overall in-hospital mortality rate showed an important increase from 0.37 to 0.98 $(\mathrm{p}=0.037)$. Also LOS decreased significantly $(-18 \%)$ from 9.8 to 8 days $(p<0.001) .73 \%$ of patients were overall admitted by surgical units with a steady increase for admission in medical ones from $22 \%$ to $34 \%$ and in particular in GE units where admission rose from $7 \%$ to $22 \%$ of overall admission of Medical Area. From the comparison between GE units and other medical area admissions, emerged that patients admitted in GE units have a not significantly lower $(p>0.05)$ NFS rate (3.5\%Vs.4.1\%) and in-hospital mortality (0.3\%Vs.1.4\%), showing a significative lower LOS $(7.1 \pm 2.2$ Vs. $11 \pm 3.6 ; \mathrm{p}<0.001)$.

Conclusions Results suggest that ACD is an emerging heathcare problem related to the rising of age. Through the years the NFS rate was stable with an increase of mortality. In this scenary GE units seem to be more efficiently obtaining a significative reduction of the cost management of ACD.

\begin{tabular}{|c|c|c|c|c|c|}
\hline Area & Total & $\begin{array}{l}\% \\
\text { distrib }\end{array}$ & $\begin{array}{l}\% \\
\text { NFS }\end{array}$ & LOS & $\begin{array}{l}\% \\
\text { death }\end{array}$ \\
\hline Surgery & 24323 & $73 \%$ & $25 \%$ & 8,3 & $0,5 \%$ \\
\hline Medical & 8893 & $27 \%$ & $4 \%$ & 10,2 & $1,1 \%$ \\
\hline Intensive Therapy & 33 & $0,1 \%$ & $48 \%$ & 22,8 & $24,2 \%$ \\
\hline Total & 33249 & $100 \%$ & $19 \%$ & 8,8 & $0,6 \%$ \\
\hline $\begin{array}{l}\text { Medical area } \\
\text { only }\end{array}$ & Total & $\begin{array}{l}\% \\
\text { distrib }\end{array}$ & & & \\
\hline Gastro units & 1743 & $20 \%$ & $3,5 \%$ & 7,1 & $0,3 \%$ \\
\hline No Gastro units & 7150 & $80 \%$ & $4,1 \%$ & 11,0 & $1,4 \%$ \\
\hline
\end{tabular}

